\title{
Effects of Ertugliflozin on Liver Enzymes in Patients with Type 2 Diabetes: A Post-Hoc Pooled Analysis of Phase 3 Trials
}

\author{
Silvina Gallo • Roberto A. Calle • Steven G. Terra • Annpey Pong • \\ Lisa Tarasenko • Annaswamy Raji (D)
}

Received: April 29, 2020 / Published online: July 9, 2020

(C) The Author(s) 2020

\begin{abstract}
Introduction: This post hoc exploratory analysis examined the effects of ertugliflozin on liver enzymes in patients with type 2 diabetes mellitus (T2DM).

Methods: Data were pooled from seven randomized, double-blind VERTIS phase 3 trials that evaluated ertugliflozin (5 $\mathrm{mg}$ and $15 \mathrm{mg}$ ) versus nonertugliflozin (placebo, glimepiride, or sitagliptin) treatment in patients with T2DM. Change from baseline at week 52 of treatment in alanine and aspartate aminotransferase (ALT and AST, respectively) serum levels (overall and categorized into tertiles by baseline ALT and AST), Fibrosis-4 Index (FIB-4), glycated hemoglobin (HbA1c), and body weight were evaluated, along with the association
\end{abstract}

Digital Features To view digital features for this article go to: https://doi.org/10.6084/m9.figshare.12481244.

S. Gallo

Pfizer Pharma GmbH, Berlin, Germany

R. A. Calle

Pfizer Inc., Cambridge, MA, USA

S. G. Terra

Pfizer Inc., Andover, MA, USA

A. Pong · A. Raji ( $\bowtie)$

Merck \& Co., Inc., Kenilworth, NJ, USA

e-mail: annaswamy.raji@merck.com

L. Tarasenko

Pfizer Inc., New York, NY, USA between changes in ALT and AST and changes in HbA1c and body weight by treatment.

Results: Baseline characteristics were balanced across treatment groups (ertugliflozin $5 \mathrm{mg}$, $n=1716$; ertugliflozin $15 \mathrm{mg}, n=1693$; non-ertugliflozin, $n=1450$ ). At week 52 of treatment, serum levels of ALT and AST were reduced in patients in the ertugliflozin treatment groups (5 and $15 \mathrm{mg}$, respectively) compared with those in the non-ertugliflozin group. The comparator-adjusted mean (95\% confidence interval [CI]) difference in change from baseline at week 52 for ALT was $-3.35(-4.40,-2.31)$ IU/L for ertugliflozin $5 \mathrm{mg}$ and $-4.08(-5.13,-3.03)$ IU/L for ertugliflozin $15 \mathrm{mg}$; for AST, the respective values were $-1.81(-2.50,-1.11) \mathrm{IU} / \mathrm{L}$ and $-2.12(-2.82$, $-1.42) \mathrm{IU} / \mathrm{L}$. The effects of ertugliflozin were detected across all baseline ALT and AST tertiles, with the highest tertile showing the greatest treatment differences. No meaningful differences were observed between treatment groups for FIB- 4 . Changes in ALT and AST showed a weak but statistically significant association with changes in HbA1c and body weight in all treatment groups.

Conclusions: Treatment with ertugliflozin resulted in a reduction in the levels of hepatic transaminases compared with the non-ertugliflozin group after 52 weeks of treatment. Changes in body weight and HbA1c contributed at least in part to the effects of ertugliflozin on liver enzymes.

Trial Registration: Clinicaltrials.gov registry numbers: NCT02033889, NCT01958671, 
NCT02036515, NCT01986855, NCT02099110, NCT02226003, NCT01999218.

Keywords: Alanine aminotransferase; Aspartate aminotransferase; Body weight; Ertugliflozin; Fibrosis-4 index; HbA1c; Liver enzymes; SGLT2 inhibitor; Type 2 diabetes mellitus

\section{Key Summary Points}

Why carry out this study?

A frequently underappreciated comorbidity of type 2 diabetes mellitus (T2DM) is non-alcoholic fatty liver disease (NAFLD). Sodium-glucose cotransporter 2 (SGLT2) inhibitors have been shown to reduce serum levels of alanine and aspartate aminotransferase (ALT and AST, respectively) in patients with T2DM with and without NAFLD.

The aim of this pooled, post hoc analysis of seven randomized, double-blind phase 3 VERTIS trials was to assess the impact of ertugliflozin on liver enzymes in patients with T2DM.

\section{What was learned from the study?}

Ertugliflozin reduced serum ALT and AST levels after 52 weeks of treatment compared with the non-ertugliflozin treatment group (placebo, glimepiride, sitagliptin), similar to results obtained with other SGLT2

inhibitors. The reduction was greatest in patients with higher baseline ALT and AST levels. No significant differences were observed between treatment groups in the Fibrosis-4 Index.

Changes in ALT and AST showed a weak but statistically significant association with changes in glycated hemoglobin (HbA1c) and body weight in all treatment groups at week 52, suggesting that these changes in hepatic transaminases are partially accounted for by reductions in body weight and $\mathrm{HbA1c}$.

\section{INTRODUCTION}

A frequently underappreciated comorbidity of type 2 diabetes mellitus (T2DM) is non-alcoholic fatty liver disease (NAFLD), with a global prevalence of $55.5 \%$ among patients with T2DM [1]. Patients with NAFLD are usually asymptomatic and are often presumptively identified through increased serum levels of alanine transaminase (ALT) and aspartate transaminase (AST) [2, 3]. In a subset of patients, NAFLD can progress to non-alcoholic steatohepatitis (NASH) and fibrosis, leading to adverse liver outcomes, including mortality, and a greater need for liver transplantations. Underscoring the importance of early diagnosis, the risk of progression to NASH appears to be greater in patients with T2DM $[4,5]$.

Sodium-glucose cotransporter 2 (SGLT2) inhibitors are an important addition to approved therapies for T2DM $[6,7]$. They have an insulinindependent mode of action and reduce renal glucose reabsorption, thereby enhancing urinary glucose excretion and reducing plasma glucose and glycated hemoglobin (HbA1c) levels [8]. The SGLT2 inhibitor drug class is associated with a reduction in blood pressure and body weight $[9,10]$. In addition, studies have shown that treatment with SGLT2 inhibitors reduces the serum levels of ALT and AST, as found with canagliflozin and dapagliflozin in patients with T2DM $[11,12]$, with empagliflozin in patients with T2DM with or without NAFLD $[13,14]$, and with dapagliflozin in patients with T2DM and NAFLD or NASH [15-17]. Furthermore, there is some evidence that SGLT2 inhibitors (empagliflozin and dapagliflozin) may improve markers of hepatic injury, hepatic steatosis, and liver fibrosis in patients with T2DM and NAFLD or NASH [13, 15-17].

Ertugliflozin, a selective inhibitor of SGLT2, is approved in the USA, European Union, and other countries for the treatment of adults with T2DM. The VERTIS (eValuation of ERTugliflozin effIcacy and Safety) phase 3 clinical program has demonstrated the efficacy and safety of oral, once-daily 5 and $15 \mathrm{mg}$ ertugliflozin, respectively, either as monotherapy or in combination with other antihyperglycemic agents [18-25]. In the post hoc analysis reported here, 
we explored the effects of ertugliflozin on ALT, AST, and the Fibrosis-4 Index (FIB-4; a non-invasive marker of hepatic fibrosis) and examined their association with changes in glycemic control (HbA1c) and body weight.

\section{METHODS}

\section{Study Design and Patient Population}

This post hoc, exploratory analysis used a pooled data set comprising seven randomized, doubleblind VERTIS phase 3 trials that evaluated the efficacy and safety of ertugliflozin compared with non-ertugliflozin (placebo, glimepiride, or sitagliptin) treatment (Table 1) [18-24]. In these studies, patients aged $\geq 18$ years with inadequately controlled T2DM per the American Diabetes Association criteria and baseline HbA1c of $7.0-11 \%$ were randomized to treatment with ertugliflozin $5 \mathrm{mg}$, ertugliflozin $15 \mathrm{mg}$, or nonertugliflozin treatment for 26-52 weeks (Table 1). Glycemic rescue therapy was prescribed for patients who exceeded protocol-specified glycemic thresholds. Patients were excluded from these studies if the ALT or AST level was $>2 \times$ the upper limit of normal at screening. Laboratory assessments were performed by one central laboratory with the following limits of normal: ALT female: $\leq 33 \mathrm{IU} / \mathrm{L} ;$ ALT male: $\leq 41 \mathrm{IU} / \mathrm{L}$, AST female: $\leq 31 \mathrm{IU} / \mathrm{L}$, AST male: $\leq 37 \mathrm{IU} / \mathrm{L}$.

\section{Endpoint and Assessments}

Endpoints were change from baseline to week 52 of treatment in serum levels of ALT and AST, and FIB-4. The association between changes in ALT and AST and changes in HbA1c and body weight were also assessed.

FIB-4 was calculated using the following equation [30, 31]:

$$
\text { FIB }-4=\frac{(\text { Age years }) \times(\text { AST U } / \mathrm{L})}{\left(\text { Platelets } 10^{9} / \mathrm{L}\right) \times \sqrt{\text { ALT U/L }}} .
$$

No additional safety assessments were evaluated in this post hoc analysis. Safety analyses relating to each study have been published previously [18-24].

\section{Statistical Analyses}

This report presents data following 52 weeks of treatment. The post hoc analyses (SAS v9.4; SAS Institute, Cary, NC, USA) examined the data at both 26 and 52 weeks, and the results were generally similar. One of the seven VERTIS studies [21] had a duration of only 26 weeks, but because the results of the analyses at 26 and 52 weeks in the other studies were similar, these data were included in the statistical analyses (as 26-week data), and new analyses without these data and based only on week 52 data were not deemed necessary.

Analyses on ALT, AST, FIB-4, HbA1c, and body weight were conducted on all randomized patients who received $\geq 1$ dose of study medication and had a baseline measurement and at least one post-baseline measurement. All analyses included data after the initiation of rescue therapy. There was no correction for type 1 error with multiple testing; the $p$ values provided from the analysis model should be considered nominal.

Change from baseline in ALT, AST, FIB-4, HbA1c, and body weight at week 52 were compared between treatment groups using a repeatedmeasures analysis of covariance (ANCOVA) model with fixed effects for treatment, time, trial, baseline value of the response variable, and the interaction of time by treatment. Time was fitted as a categorical term. This analysis was also conducted on pooled sets of patients grouped into tertiles according to baseline levels of ALT and AST.

The association between changes in ALT and AST and changes in HbA1c and body weight by treatment was assessed by Pearson correlation by treatment. In addition, a repeated-measures ANCOVA model including body weight and HbA1c change from baseline, with the baseline value of the response variables as covariates and with fixed effects for treatment, time, trial, baseline value of the response variable, and the interaction of time by treatment, was performed to assess the contributions of weight loss and improvements in glycemic control to the changes in ALT and AST at week 52. 


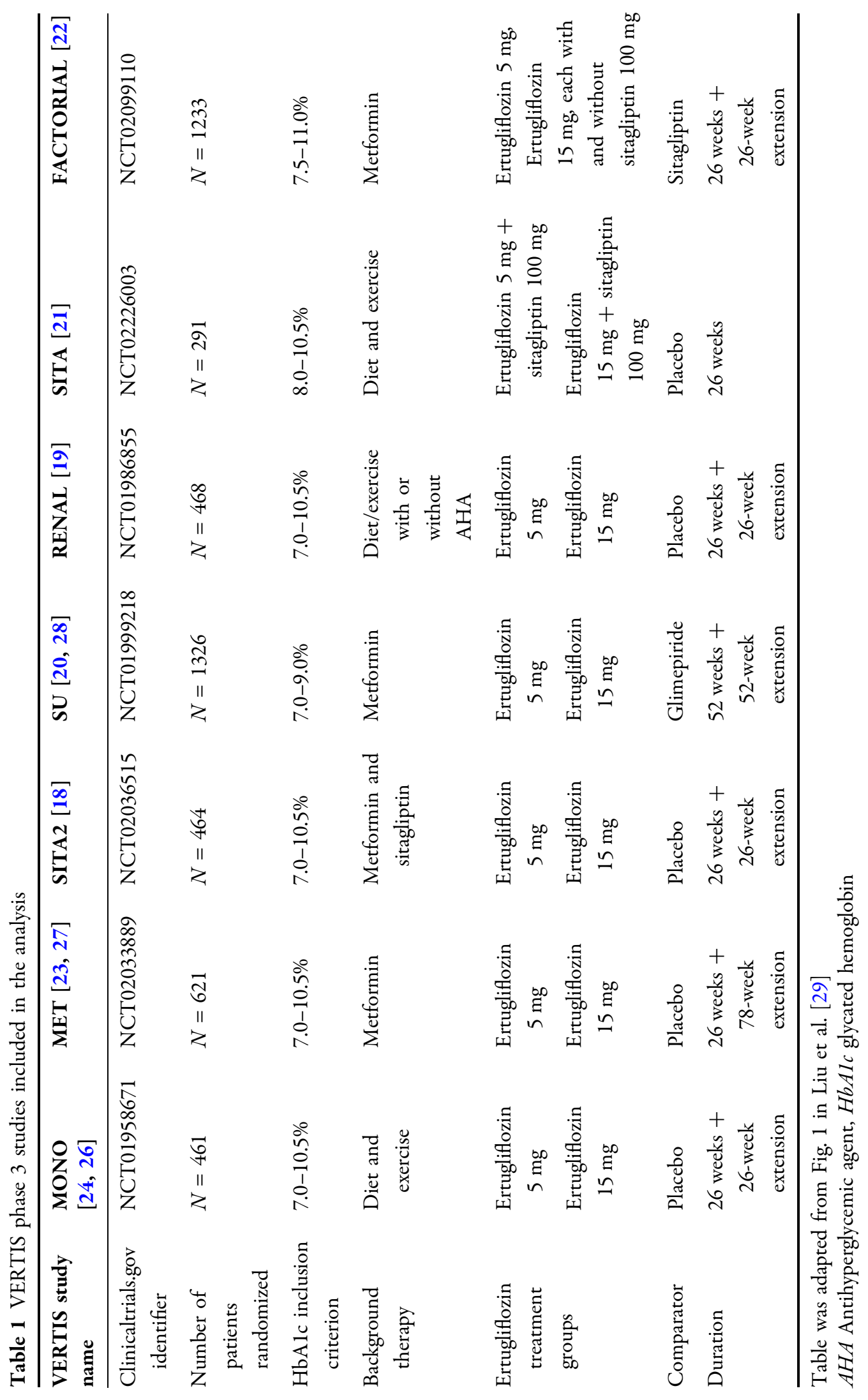


Table 2 Baseline demographics and disease characteristics of all patients included in the analysis

\begin{tabular}{|c|c|c|c|}
\hline \multirow{2}{*}{$\begin{array}{l}\text { Baseline demographics and disease } \\
\text { characteristics }\end{array}$} & \multicolumn{3}{|l|}{ Treatment group } \\
\hline & $\begin{array}{l}\text { Non-ertugliflozin } \\
(n=1450)\end{array}$ & $\begin{array}{l}\text { Ertugliflozin } 5 \mathrm{mg} \\
(n=1716)\end{array}$ & $\begin{array}{l}\text { Ertugliflozin } 15 \mathrm{mg} \\
(n=1693)\end{array}$ \\
\hline Age (years) & $57.8(10.3)$ & $57.9(10.2)$ & $57.8(10.2)$ \\
\hline Male, $n(\%)$ & $787(54.3 \%)$ & $885(51.6 \%)$ & $844(49.9 \%)$ \\
\hline Body weight $(\mathrm{kg})^{\mathrm{a}}$ & $89.2(21.3)$ & $89.1(20.8)$ & $87.5(19.3)$ \\
\hline BMI $\left(\mathrm{kg} / \mathrm{m}^{2}\right)$ & $31.6(6.3)$ & $31.9(6.1)$ & $31.5(5.8)$ \\
\hline Duration of T2DM (years) & $7.8(6.3)$ & $7.9(6.5)$ & $8.0(6.3)$ \\
\hline HbAlc $(\%)^{b}$ & $8.1(0.9)$ & $8.2(0.9)$ & $8.2(0.9)$ \\
\hline $\operatorname{ALT}(\mathrm{IU} / \mathrm{L})^{\mathrm{c}}$ & $26.6(14.0)$ & $27.4(14.6)$ & $27.6(16.1)$ \\
\hline $\operatorname{AST}(\mathrm{IU} / \mathrm{L})^{\mathrm{d}}$ & $21.7(9.4)$ & $22.0(9.8)$ & $22.3(10.4)$ \\
\hline FIB- $4^{e}$ & $1.12(0.56)$ & $1.11(0.57)$ & $1.10(0.53)$ \\
\hline Platelet count $\left(10^{9} / \mathrm{L}\right)^{\mathrm{f}}$ & $239.2(61.2)$ & $241.6(60.4)$ & $245.1(62.0)$ \\
\hline
\end{tabular}

Data are presented as the mean with the standard deviation (SD) in parenthesis, unless otherwise specified $A L T$ Alanine aminotransferase, AST aspartate aminotransferase, BMI body mass index, FIB-4 Fibrosis-4 Index, HbAIc glycated hemoglobin, $T 2 D M$ type 2 diabetes mellitus

${ }_{a-f} n$ is the number of patients in the non-ertugliflozin, ertugliflozin $5 \mathrm{mg}$, and ertugliflozin $15 \mathrm{mg}$ treatment arms, respectively. ${ }^{\mathrm{a}} n=1442, n=1706$, and $n=1686 ;{ }^{\mathrm{b}} n=1439, n=1695$, and $n=1679 ;{ }^{\mathrm{c}} n=1417, n=1677$, and $n=1664 ;{ }^{\mathrm{d}} n=1415, n=1672$, and $n=1664 ;{ }^{\mathrm{e}} n=1377, n=1626$, and $n=1621 ;{ }^{\mathrm{f}} n=1407, n=1657$, and $n=1651$

\section{Statement of Ethics Compliance}

Each of the seven studies included in the analysis was conducted in compliance with the ethical principles of the Declaration of Helsinki and in compliance with all International Conference on Harmonization Good Clinical Practice Guidelines. The final protocols and informed consent documentation were reviewed and approved by the Institutional Review Boards or Independent Ethics Committees at each investigational center. Written informed consent was obtained from all participants.

\section{RESULTS}

\section{Patient Disposition and Baseline Characteristics}

A total of 4859 patients were included in the final analysis (ertugliflozin $5 \mathrm{mg}, n=1716$; ertugliflozin $15 \mathrm{mg}, n=1693$; non-ertugliflozin, $n=1450$ ). Patients had a mean (standard deviation [SD]) duration of T2DM of 7.9 (6.4) years and a mean age of 57.8 (10.2) years; baseline characteristics were similar across the three treatment groups (Table 2).

\section{Serum Levels of ALT and AST}

Treatment with ertugliflozin $5 \mathrm{mg}$ and $15 \mathrm{mg}$ was associated with a decrease in serum levels of ALT and AST from baseline at week 52 compared with the non-ertugliflozin treatment, which showed increases in ALT and AST. Both the absolute and percentage decrease from baseline were greater with ertugliflozin $5 \mathrm{mg}$ and $15 \mathrm{mg}$ than with non-ertugliflozin treatment (Fig. 1).

Patients were categorized into tertiles according to baseline serum levels of ALT and AST as follows:

ALT: $\leq 19,>19$ to $\leq 29$, and $>29 \mathrm{IU} / \mathrm{L}$;

AST: $\leq 17,>17$ to $\leq 23$, and $>23 \mathrm{IU} / \mathrm{L}$. 
a

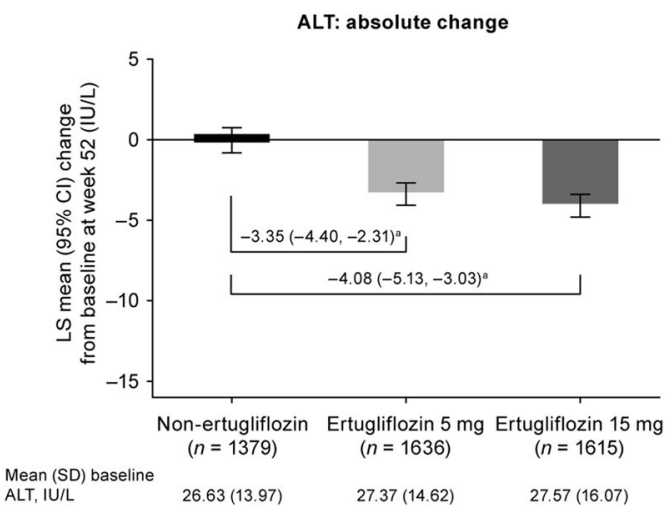

b

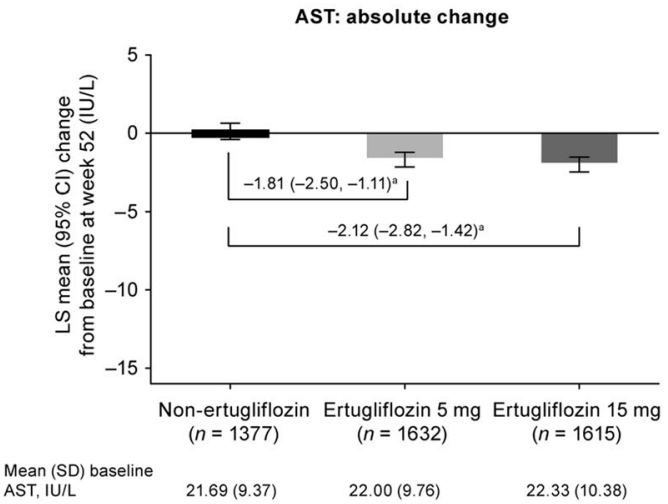

Fig. 1 Least squares $(L S)$ mean absolute and percentage change from baseline at week 52 in alanine aminotransferase $(A L T)(\mathbf{a})$ and aspartate aminotransferase $(A S T)$

Across all tertiles, ertugliflozin $5 \mathrm{mg}$ and $15 \mathrm{mg}$ provided a greater benefit (reduction) on ALT and AST levels than did the non-ertugliflozin treatment at week 52 (Fig. 2). For both ertugliflozin doses, the largest reduction from baseline was observed in the highest tertile. For the highest baseline ALT tertile (> $29 \mathrm{IU} / \mathrm{L})$, ertugliflozin $5 \mathrm{mg}$ and $15 \mathrm{mg}$ provided a least squares (LS) mean (95\% confidence interval [CI]) reduction from baseline of $-10.69(-12.51,-8.88)$ IU/L and -11.02 $(-12.82,-9.23) \mathrm{IU} / \mathrm{L}$, respectively, which is more than twice the reduction shown in the non-ertugliflozin treatment arm (LS mean $-4.19 \mathrm{IU} / \mathrm{L} ; 95 \% \mathrm{CI}-6.21,-2.17)$. For the highest baseline AST tertile (>23 IU/L), ertugliflozin $5 \mathrm{mg}$ and $15 \mathrm{mg}$ provided a LS mean (95\% CI) reduction from baseline of -6.79 $(-7.98,-5.60)$ IU/L and $-7.17(-8.33$, - 6.01) IU/L, respectively; LS mean change

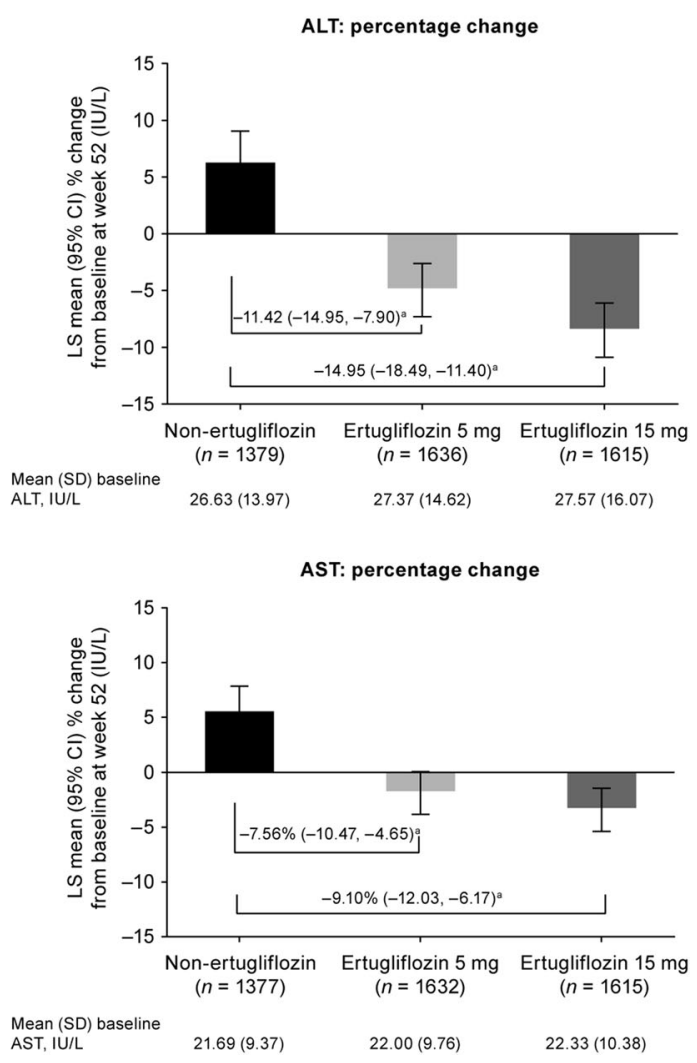

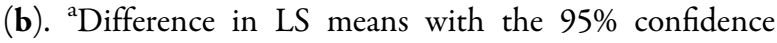
interval $(C I)$ in parenthesis. SD Standard deviation

from baseline in the non-ertugliflozin arm was $-4.32(-5.64,-3.01) \mathrm{IU} / \mathrm{L}$.

\section{FIB-4}

At week 52, the mean (SD) FIB-4 value was 1.11 (0.51) for the ertugliflozin $5 \mathrm{mg}$ group, 1.09 (0.55) for the ertugliflozin $15 \mathrm{mg}$ group, and $1.16(0.52)$ for the non-ertugliflozin group. There were no meaningful differences in absolute LS mean change from baseline at week 52 in FIB-4 between the treatment groups (Table 3).

\section{Association of ALT and AST With Reductions in HbA1c and Body Weight}

At week 52, both doses of ertugliflozin led to greater reductions from baseline in HbA1c compared with the non-ertugliflozin group 

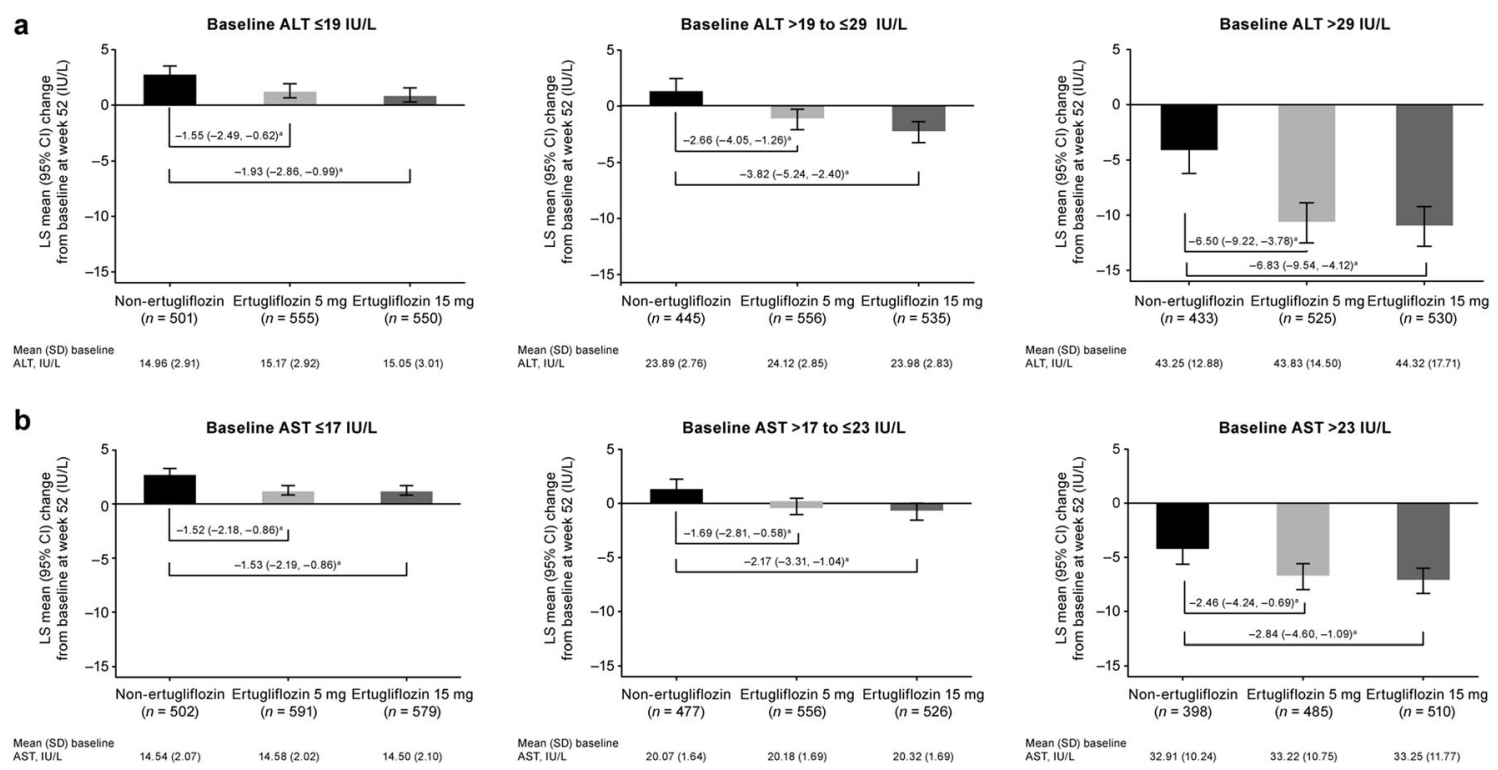

Fig. 2 Least squares $(L S)$ mean absolute change from baseline at week 52 in ALT (a) and AST (b) by tertiles of baseline ALT and AST. ${ }^{a}$ Difference in LS means with the

95\% CI in parenthesis. ALT Alanine aminotransferase, $A S T$ aspartate aminotransferase, $C I$ confidence interval, $S D$ standard deviation

Table 3 Least squares mean absolute change from baseline at week 52 in Fibrosis-4 Index

\begin{tabular}{lllll}
\hline Treatment arms & Baseline FIB-4 & Week 52 FIB-4 & \multicolumn{2}{l}{ Change in FIB-4 from baseline at week 52 } \\
\cline { 4 - 5 } & Mean (SD) & Mean (SD) & LS mean (95\% CI) & $\begin{array}{l}\text { Difference in LS mean relative to } \\
\text { non-ertugliflozin (95\% CI) }\end{array}$ \\
\hline Non-ertugliflozin & $1.12(0.56)$ & $1.16(0.52)$ & $0.01(-0.02,0.03)$ & - \\
Ertugliflozin $5 \mathrm{mg}$ & $1.11(0.57)$ & $1.11(0.51)$ & $0.02(0.00,0.04)$ & $0.01(-0.02,0.04)$ \\
Ertugliflozin $15 \mathrm{mg}$ & $1.10(0.53)$ & $1.09(0.55)$ & $0.01(-0.01,0.03)$ & $0.00(-0.03,0.03)$ \\
\hline
\end{tabular}

$C I$ Confidence interval, $L S$ least squares, FIB-4 Fibrosis-4 Index, SD standard deviation

(Fig. 3). Ertugliflozin also led to reductions from baseline in body weight, in contrast to the nonertugliflozin group, which showed no overall change from baseline (Fig. 3).

The changes in ALT and AST from baseline to week 52 were associated with changes in HbA1c and body weight in all treatment groups, with $r$ values ranging from 0.12 to 0.16 for $\mathrm{HbA1c}$ and from 0.13 to 0.23 for body weight (all $p \leq$ 0.0002).

With the addition of body weight and HbA1c as covariates in the statistical model, both doses of ertugliflozin remained more effective than non-ertugliflozin therapies in reducing serum levels of ALT and AST at week 52 (Table 4).
However, the LS mean differences between treatment groups (ertugliflozin vs. non-ertugliflozin) when body weight and HbA1c were included as covariates (Table 4) were smaller than those obtained when body weight and HbA1c were not included as covariates (Fig. 1).

\section{DISCUSSION}

In this post hoc pooled analysis of patients with T2DM, treatment with ertugliflozin $5 \mathrm{mg}$ and $15 \mathrm{mg}$ reduced serum levels of ALT and AST after 52 weeks of treatment compared with nonertugliflozin treatment. When patients were 

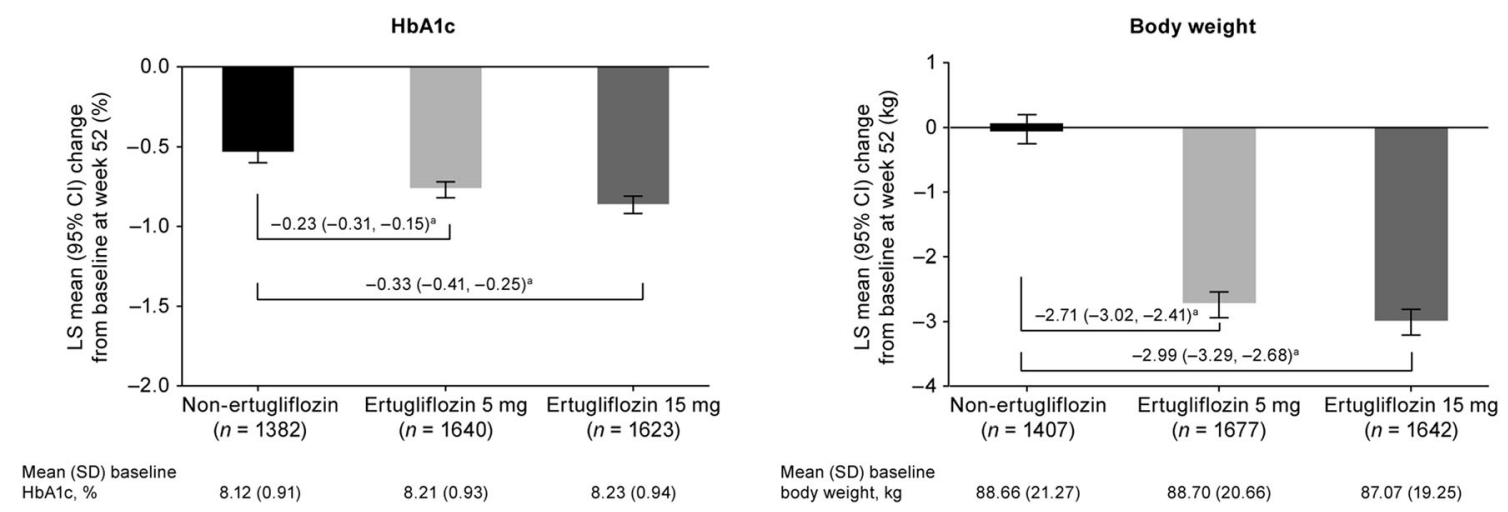

Fig. 3 Least squares $(L S)$ mean change from baseline at week 52 in glycated hemoglobin $(H b A 1 c)$ and body weight. ${ }^{a}$ Difference in LS means ( $\left.95 \% \mathrm{CI}\right)$. CI confidence interval, SD standard deviation

Table 4 Treatment difference in least squares mean absolute and percentage change from baseline to week 52 in alanine and aspartate transaminases (repeated-measures analysis with body weight and glycated hemoglobin as covariates)

\begin{tabular}{lll}
\hline Treatment & \multicolumn{1}{l}{ Difference in $\mathbf{L S}$ mean change from baseline relative to non-ertugliflozin $\mathbf{9 5 \%} \mathbf{C I})$} \\
\cline { 2 - 3 } & Absolute change, IU/L & Percentage change \\
\hline ALT & $-2.35(-3.38,-1.33)$ & $-8.08(-11.71,-4.44)$ \\
Ertugliflozin $5 \mathrm{mg}$ & $-2.93(-3.96,-1.90)$ & $-10.77(-14.42,-7.11)$ \\
Ertugliflozin $15 \mathrm{mg}$ & $-1.37(-2.06,-0.68)$ & $-5.75(-8.84,-2.66)$ \\
AST & $-1.61(-2.30,-0.92)$ & $-7.07(-10.17,-3.96)$ \\
Ertugliflozin $5 \mathrm{mg}$ & Ertugliflozin $15 \mathrm{mg}$ &
\end{tabular}

$A L T$ Alanine aminotransferase, $A S T$ aspartate aminotransferase, $C I$ confidence interval, $H b A 1 c$ glycated hemoglobin, $L S$ least squares

grouped by baseline ALT and AST tertiles, the greatest reduction from baseline was seen in the highest tertile for ALT $(>29 \mathrm{IU} / \mathrm{L})$ and AST ( $>23 \mathrm{IU} / \mathrm{L}$ ), and was more prominent for ALT. Given that these clinical trials excluded patients with baseline ALT or AST $>2 \times$ upper limit of normal, the potential effect of ertugliflozin in reducing hepatic transaminases may be underestimated-especially for patient groups with higher transaminase levels, as found in some patients with NAFLD. Overall, these findings are consistent with those reported for other SGLT2 inhibitors in patients with T2DM $[11,12,14]$.

The present analysis also assessed the impact of ertugliflozin on liver fibrosis using the FIB-4, a calculated score that has been shown to correlate with the stage of fibrosis (by histologic status) in patients with NAFLD [32]. The FIB-4 scores observed in this study at baseline and at week 52 likely correspond to fibrosis stage 0-2 [32], and are consistent with previously published FIB-4 scores in patients with T2DM and no diagnosis of liver disease [12]. No difference between treatment groups in the change from baseline to week 52 in FIB-4 score was observed. This lack of effect in patients receiving ertugliflozin may be due to the patient population enrolled; there was no requirement for liver disease, thereby resulting in low levels of baseline fibrosis. In studies of patients with T2DM 
and NAFLD $[33,34]$ or biopsy-confirmed NASH [35] treated with SGLT2 inhibitors, on average, baseline FIB-4 scores (mean values were 1.42 and 2.08 in two studies; median value was 1.75 in another study) were higher than those in the present analysis, suggesting that at least some patients in those studies may fall within fibrosis histology stage 3-4 [32]. In those studies, improvements in the FIB- 4 scores were observed following SGLT2 inhibitor treatment.

Changes in ALT and AST showed a weak but statistically significant association with changes in HbA1c and body weight in all treatment groups at week 52 . When body weight and HbA1c were added as covariates in the repeatedmeasures ANCOVA model, the effect of ertugliflozin versus non-ertugliflozin on ALT and AST remained, but the effect sizes were smaller than those from the analysis without these factors as covariates. This suggests that changes in HbA1c and body weight may partly contribute to the effects of ertugliflozin on aminotransferases, but do not account entirely for the observed reductions in this post hoc analysis. Relationships between changes in liver enzymes and changes in body weight or HbA1c may not be linear, and this could account for the weak correlation between the parameters.

Similar analyses with other SGLT2 inhibitors have produced divergent findings. In a pooled analysis of patients with T2DM treated with canagliflozin, the significant reductions in ALT and AST at week 26 were fully attributed to lowered body weight and HbA1c level [11]. However, in a pooled analysis of patients treated with empagliflozin, the significant reductions in ALT after 28 weeks of treatment were found to be largely independent of changes in body weight and glycemic control [14]. Similarly, in a clinical study of ipragliflozin in patients with T2DM-associated liver dysfunction, changes in serum ALT levels did not correlate with changes in body weight [36]. The reason for these discrepancies is unclear but may be related to methodologic and patient population differences among studies and their associated statistical models for pooled analyses.

There are limitations associated with this analysis. The post hoc nature of the analysis, the exclusion of patients with elevated levels of
ALT and AST from the studies in this analysis, and the heterogeneous non-ertugliflozin control group could be considered potential limitations. Further, the analysis was not conducted in patients specifically with any chronic liver disease, such as NAFLD or NASH. Lastly, there was no correction for type 1 error with multiple testing.

\section{CONCLUSION}

In this post hoc, exploratory analysis, ertugliflozin was shown to be associated with reductions in hepatic transaminases in patients treated for 52 weeks compared with the group receiving non-ertugliflozin treatment (placebo, glimepiride, or sitagliptin). Weak correlations were observed with body weight and HbA1c. No differences in the FIB-4 score were observed. Prospective and mechanistic studies are needed to assess the effects of ertugliflozin and other SGLT2 inhibitors on liver enzymes in patients with T2DM with and without NAFLD.

\section{ACKNOWLEDGEMENTS}

The authors would like to thank all study participants, physicians, investigators, and staff at the participating study centers for their contributions to the individual studies.

Funding. This study was sponsored by Merck Sharp \& Dohme Corp., a subsidiary of Merck \& Co., Inc., Kenilworth, NJ, USA (MSD), in collaboration with Pfizer Inc., New York, NY, USA. The Rapid Service Fee was funded by MSD in collaboration with Pfizer Inc., New York, NY, USA.

Medical Writing Assistance. Medical writing support was provided by Diane Hoffman, PhD, of Engage Scientific Solutions and was funded by MSD and Pfizer Inc.

Authorship. All authors meet the International Committee of Medical Journal Editors (ICMJE) criteria for authorship for this article, take responsibility for the integrity of the work 
as a whole, and have given their approval for this version to be published.

Prior Presentation. Some of these data were presented at the 55th Annual Meeting of the European Association for the Study of Diabetes (EASD), September 16-20, 2019, Barcelona, Spain, and the American Association for the Study of Liver Diseases (AASLD)/The Liver Meeting 2019, November 8-12, 2019, Boston, MA, USA.

Disclosures. Annpey Pong and Annaswamy Raji are employees of MSD and may own stock in Merck \& Co., Inc., Kenilworth, NJ, USA. Silvina Gallo, Roberto A. Calle, Steven G. Terra, and Lisa Tarasenko are employees of Pfizer Inc. and may hold stock and/or stock options in Pfizer Inc.

Compliance with Ethics Guidelines. Each of the seven studies included in the analysis was conducted in compliance with the ethical principles of the Declaration of Helsinki and in compliance with all International Conference on Harmonisation Good Clinical Practice Guidelines. The final protocols and informed consent documentation were reviewed and approved by the Institutional Review Boards or Independent Ethics Committees at each investigational center. Written informed consent was obtained from all participants.

Data Availability. Upon request, and subject to certain criteria, conditions, and exceptions (see https://www.pfizer.com/science/ clinical-trials/trial-data-and-results for more information), Pfizer will provide access to individual de-identified participant data from Pfizersponsored global interventional clinical studies conducted for medicines, vaccines, and medical devices (1) for indications that have been approved in the USA and/or EU or (2) in programs that have been terminated (i.e., development for all indications has been discontinued). Pfizer will also consider requests for the protocol, data dictionary, and statistical analysis plan. Data may be requested from Pfizer trials 24 months after study completion. The de-identified participant data will be made available to researchers whose proposals meet the research criteria and other conditions, and for which an exception does not apply, via a secure portal. To gain access, data requestors must enter into a data access agreement with Pfizer.

Open Access. This article is licensed under a Creative Commons Attribution-NonCommercial 4.0 International License, which permits any non-commercial use, sharing, adaptation, distribution and reproduction in any medium or format, as long as you give appropriate credit to the original author(s) and the source, provide a link to the Creative Commons licence, and indicate if changes were made. The images or other third party material in this article are included in the article's Creative Commons licence, unless indicated otherwise in a credit line to the material. If material is not included in the article's Creative Commons licence and your intended use is not permitted by statutory regulation or exceeds the permitted use, you will need to obtain permission directly from the copyright holder. To view a copy of this licence, visit http://creativecommons.org/licenses/by$\mathrm{nc} / 4.0 /$.

\section{REFERENCES}

1. Younossi ZM, Golabi P, de Avila L, Paik JM, Srishord $\mathrm{M}$, Fukui $\mathrm{N}$, et al. The global epidemiology of NAFLD and NASH in patients with type 2 diabetes: a systematic review and meta-analysis. J Hepatol. 2019;71:793-801.

2. Tomic D, Kemp WW, Roberts SK. Nonalcoholic fatty liver disease: current concepts, epidemiology and management strategies. Eur J Gastroenterol Hepatol. 2018;30:1103-15.

3. Spengler EK, Loomba R. Recommendations for diagnosis, referral for liver biopsy, and treatment of nonalcoholic fatty liver disease and nonalcoholic steatohepatitis. Mayo Clin Proc. 2015;90:1233-46.

4. Anstee QM, Targher G, Day CP. Progression of NAFLD to diabetes mellitus, cardiovascular disease or cirrhosis. Nat Rev Gastroenterol Hepatol. 2013;10:330-44.

5. Cotter TG, Rinella M. Nonalcoholic fatty liver disease 2020: The state of the disease. Gastroenterology. 2020;158:1851-64. 
6. Cosentino F, Grant PJ, Aboyans V, et al. 2019 ESC Guidelines on diabetes, pre-diabetes, and cardiovascular diseases developed in collaboration with the EASD. Eur Heart J. 2020;41:255-323.

7. American Diabetes Association. 9. Pharmacologic approaches to glycemic treatment: standards of medical care in diabetes-2019. Diabetes Care. 2019;42:S90-102.

8. Chao EC. SGLT-2 inhibitors: a new mechanism for glycemic control. Clin Diabetes. 2014;32:4-11.

9. Wang H, Yang J, Chen X, Qiu F, Li J. Effects of sodium-glucose cotransporter 2 inhibitor monotherapy on weight changes in patients with type 2 diabetes mellitus: a bayesian network metaanalysis. Clin Ther. 2019;41(322-34):e11.

10. Mazidi M, Rezaie P, Gao HK, Kengne AP. Effect of sodium-glucose cotransport- 2 inhibitors on blood pressure in people with type 2 diabetes mellitus: a systematic review and meta-analysis of 43 randomized control trials with 22528 patients. J Am Heart Assoc. 2017;6(6):e004007.

11. Leiter LA, Forst T, Polidori D, Balis DA, Xie J, Sha S. Effect of canagliflozin on liver function tests in patients with type 2 diabetes. Diabetes Metab. 2016;42:25-32.

12. Gastaldelli A, Repetto E, Guja C, et al. Exenatide and dapagliflozin combination improves markers of liver steatosis and fibrosis in patients with type 2 diabetes. Diabetes Obes Metab. 2020;22:393-403.

13. Kuchay MS, Krishan S, Mishra SK, et al. Effect of empagliflozin on liver fat in patients with type 2 diabetes and nonalcoholic fatty liver disease: a randomized controlled trial (e-lift trial). Diabetes Care. 2018;41:1801-8.

14. Sattar N, Fitchett D, Hantel S, George JT, Zinman B. Empagliflozin is associated with improvements in liver enzymes potentially consistent with reductions in liver fat: results from randomised trials including the EMPA-REG OUTCOME(R) trial. Diabetologia. 2018;61:2155-63.

15. Shimizu M, Suzuki K, Kato K, et al. Evaluation of the effects of dapagliflozin, a sodium-glucose co-transporter- 2 inhibitor, on hepatic steatosis and fibrosis using transient elastography in patients with type 2 diabetes and non-alcoholic fatty liver disease. Diabetes Obes Metab. 2019;21:285-92.

16. Tobita H, Sato S, Miyake T, Ishihara S, Kinoshita Y. Effects of dapagliflozin on body composition and liver tests in patients with nonalcoholic steatohepatitis associated with type 2 diabetes mellitus: a prospective, open-label, uncontrolled study. Curr Ther Res Clin Exp. 2017;87:13-9.
17. Eriksson JW, Lundkvist P, Jansson PA, et al. Effects of dapagliflozin and n-3 carboxylic acids on nonalcoholic fatty liver disease in people with type 2 diabetes: a double-blind randomised placebo-controlled study. Diabetologia. 2018;61:1923-34.

18. Dagogo-Jack S, Liu J, Eldor R, et al. Efficacy and safety of the addition of ertugliflozin in patients with type 2 diabetes mellitus inadequately controlled with metformin and sitagliptin: the VERTIS SITA2 placebo-controlled randomized study. Diabetes Obes Metab. 2018;20:530-40.

19. Grunberger G, Camp S, Johnson J, et al. Ertugliflozin in patients with stage 3 chronic kidney disease and type 2 diabetes mellitus: the VERTIS RENAL randomized study. Diabetes Ther. 2018;9: 49-66.

20. Hollander P, Liu J, Hill J, et al. Ertugliflozin compared with glimepiride in patients with type 2 diabetes mellitus inadequately controlled on metformin: the VERTIS SU randomized study. Diabetes Ther. 2018;9:193-207.

21. Miller S, Krumins T, Zhou H, et al. Ertugliflozin and sitagliptin co-initiation in patients with type 2 diabetes: the VERTIS SITA randomized study. Diabetes Ther. 2018;9:253-68.

22. Pratley RE, Eldor R, Raji A, et al. Ertugliflozin plus sitagliptin versus either individual agent over 52 weeks in patients with type 2 diabetes mellitus inadequately controlled with metformin: the VERTIS FACTORIAL randomized trial. Diabetes Obes Metab. 2018;20:1111-20.

23. Rosenstock J, Frias J, Pall D, et al. Effect of ertugliflozin on glucose control, body weight, blood pressure and bone density in type 2 diabetes mellitus inadequately controlled on metformin monotherapy (VERTIS MET). Diabetes Obes Metab. 2018;20:520-9.

24. Terra SG, Focht K, Davies M, et al. Phase III, efficacy and safety study of ertugliflozin monotherapy in people with type 2 diabetes mellitus inadequately controlled with diet and exercise alone. Diabetes Obes Metab. 2017;19:721-8.

25. Ji L, Liu Y, Miao H, et al. Safety and efficacy of ertugliflozin in Asian patients with type 2 diabetes mellitus inadequately controlled with metformin monotherapy: VERTIS Asia. Diabetes Obes Metab. 2019;21:1474-82.

26. Aronson R, Frias J, Goldman A, Darekar A, Lauring B, Terra SG. Long-term efficacy and safety of ertugliflozin monotherapy in patients with inadequately controlled T2DM despite diet and exercise: VERTIS MONO extension study. Diabetes Obes Metab. 2018;20:1453-60. 
27. Gallo S, Charbonnel B, Goldman A, et al. Longterm efficacy and safety of ertugliflozin in patients with type 2 diabetes mellitus inadequately controlled with metformin monotherapy: 104-week VERTIS MET trial. Diabetes Obes Metab. 2019;21: 1027-36.

28. Hollander P, Hill J, Johnson J, et al. Results of VERTIS SU extension study: safety and efficacy of ertugliflozin treatment over 104 weeks compared to glimepiride in patients with type 2 diabetes mellitus inadequately controlled on metformin. Curr Med Res Opin. 2019;35:1335-43.

29. Liu J, Patel S, Cater NB, et al. Efficacy and safety of ertugliflozin in East/Southeast Asian patients with type 2 diabetes mellitus. Diabetes Obes Metab. 2020;22:574-82.

30. Sterling RK, Lissen E, Clumeck N, et al. Development of a simple noninvasive index to predict significant fibrosis in patients with $\mathrm{HIV} / \mathrm{HCV}$ coinfection. Hepatology. 2006;43:1317-25.

31. Vallet-Pichard A, Mallet V, Nalpas B, et al. FIB-4: an inexpensive and accurate marker of fibrosis in HCV infection. Comparison with liver biopsy and fibrotest. Hepatology. 2007;46:32-6.
32. Shah AG, Lydecker A, Murray K, Tetri BN, Contos MJ, Sanyal AJ. Comparison of noninvasive markers of fibrosis in patients with nonalcoholic fatty liver disease. Clin Gastroenterol Hepatol. 2009;7:1104-12.

33. Itani T, Ishihara T. Efficacy of canagliflozin against nonalcoholic fatty liver disease: a prospective cohort study. Obes Sci Pract. 2018;4:477-82.

34. Ohki T, Isogawa A, Toda N, Tagawa K. Effectiveness of ipragliflozin, a sodium-glucose co-transporter 2 inhibitor, as a second-line treatment for non-alcoholic fatty liver disease patients with type 2 diabetes mellitus who do not respond to incretin-based therapies including glucagon-like peptide- 1 analogs and dipeptidyl peptidase- 4 inhibitors. Clin Drug Investig. 2016;36:313-9.

35. Seko Y, Nishikawa T, Umemura A, et al. Efficacy and safety of canagliflozin in type 2 diabetes mellitus patients with biopsy-proven nonalcoholic steatohepatitis classified as stage 1-3 fibrosis. Diabetes Metab Syndr Obes. 2018;11:835-43.

36. Komiya C, Tsuchiya K, Shiba K, et al. Ipragliflozin improves hepatic steatosis in obese mice and liver dysfunction in type 2 diabetic patients irrespective of body weight reduction. PLoS One. 2016;11:e0151511. 